\title{
Radiotherapy treatment planning for breast cancer patients after a subcutaneous mastectomy with the use of a prosthesis or expander
}

\author{
Piotr Kędzierawski1,2, Krzysztof Buliński2, 3, Tomasz Kuszewski2, 3, Katarzyna Wnuk2,3, \\ Andrzej Dąbrowski2, ${ }^{2}$, Krzysztof Lis², Janusz Braziewicz ${ }^{2,3}$, Krzysztof Ślosarek ${ }^{4,5}$
}

\author{
Institute of the Health Sciences, Collegium Medicum, Jan Kochanowski University, Kielce, Poland \\ ${ }^{2}$ The Holycross Cancer Centre, Kielce, Poland \\ 3/nstitute of Physics, Collegium Medicum, Jan Kochanowski University, Kielce, Poland \\ ${ }^{4}$ Collegium Medicum, Jan Kochanowski University, Kielce, Poland \\ ${ }^{5}$ M. Sklodowska-Curie National Research Institute of Oncology, Gliwice Branch, Gliwice, Poland
}

\begin{abstract}
Introduction. Medical physicists planning radiation treatment are increasingly confronted with situations that require special attention. Undoubtedly, one such situation is the postoperative irradiation of a patient with breast cancer in which there is a prosthesis or an expander. In recent years, expanders have appeared in this location, which, due to the density of the device's valve makes it difficult to prepare an acceptable treatment plan. The study aims to present treatment planning in these situation in various Polish cancer centres and to compare overall patient preparation for the treatment.

Material and methods. A questionnaire was prepared and sent to more than 20 radiotherapy departments, which included basic questions regarding the preparation of an irradiation plan for patients treated for breast cancer after a subcutaneous mastectomy with immediate reconstruction with a prosthesis or expander. The survey encompassed eight radiotherapy departments.

Results. Not all radiotherapy departments require a manufacturer's certificate, which shows that the manufacturer does not prohibit the use of a prosthesis/expander during treatment with ionizing radiation. The X $6 \mathrm{MV}$ photons and the supine position, total and fraction doses: from 40 to $60 \mathrm{~Gy}$ and from 2 to $2.67 \mathrm{~Gy}$, respectively, are the most commonly used. The way of defining them also depends on the oncological centre. The most commonly used irradiation technique is VMAT.

Conclusion. The conclusion from the questionnaire - no standardisation of treatment planning - should encourage the community, at least medical physicists, to develop rules of conduct in this case. Irradiation techniques are mainly dynamic ones. The expander or prosthesis does not significantly affect the dose distributions.
\end{abstract}

Key words: breast cancer, subcutaneous mastectomy, prosthesis, expander, radiotherapy techniques, dose calculation algorithms

\section{Introduction}

Irradiation of patients with breast cancer is well-established. It has a positive effect on local control, but also on long-term survival, which has been proven in many clinical studies [1, 2]. The use of radiotherapy in these patients has a long tradition. The arrangement, due to the anatomy of the patients, were

\section{How to cite:}

Kędzierawski P, Buliński K, Kuszewski T, Wnuk K, Dąbrowski A, Lis K, Braziewicz J, Ślosarek K. Radiotherapy treatment planning for breast cancer patients after a subcutaneous mastectomy with the use of a prosthesis or expander. NOWOTWORY J Oncol 2021; 71: 146-152.

This article is available in open access under Creative Common Attribution-Non-Commercial-No Derivatives 4.0 International (CC BY-NC-ND 4.0) license, allowing to download articles and share them with others as long as they credit the authors and the publisher, but without permission to change them in any way or use them commercially. 
two opposite fields. At that time, this technique and an energy of $250 \mathrm{KeV}$ were used. In the 1970s, in Poland, cobalt machines started to be used in radiotherapy. In this case, the technique of "tangential" open fields was supplemented with wedge filters. Instead of two fields, there were four - two open and two with wedges. Various types of patient immobilisation systems, such as breast boards, T-holders, or vacuum mattresses, slowly began to enter clinical practice. In the first years of the use of linear accelerators, the techniques of irradiating breast cancer patients did not change much. The technique of opposite fields was still dominant. The situation did not change with the implementation of computed tomography (CT) in planning and the use of a multi-leaf collimator (MLC) $[3,4]$.

A radical change took place when planning physicists got the opportunity to plan a dynamically changing dose - intensity-modulated radiotherapy (IMRT) [5]. Slowly but surely, the technique of opposite fields was replaced by several beams with a dynamic dose change. In recent years, the dynamic arc technique (volumetric modulated arc therapy - VMAT) has been increasingly used as a technique in the irradiation of breast cancer patients [6]. The calculated dose distributions are influenced not only by beam geometry but also by algorithms used in treatment planning systems. The irradiated volume contains different anatomical structures with different densities: lung tissue, bones (ribs), and soft (glandular and fatty) tissue. While simple algorithms (based on the dose decrease as a function of depth and distance from the beam axis) give a good approximation of the dose distribution in a medium (section) of the same density; at the border of mediums of different density they completely distort the dose distribution. The currently used algorithms take into account most of the physical phenomena related to radiation absorption, therefore the calculated and measured dose distributions are consistent within the measurement uncertainty. Another problem in the irradiation of patients with breast cancer is the mobility of the chest wall and the increasingly frequent appearance of high-density implants in the irradiated volume. The presence of high-density materials can be considered in at least three aspects. There are program algorithms that calculate dose distributions that have upper-density limitations. This means that when planning treatment, it is necessary to change this value to an acceptable value for the algorithm used. This procedure causes the calculations to be inconsistent with reality. The presence of high-density material alters the energy spectrum at the volume boundary, which affects the dose. Finally, artifacts make it much harder to properly contour target volumes. Artifacts can be reduced using appropriate software or manually. Typically one calibration curve is used: HU - density [7]. The latter issue was the reason for surveying in Polish radiotherapy departments regarding the irradiation of breast cancer patients after a subcutaneous mastectomy with the use of a prosthesis or an expander.

\section{Material and methods}

\section{Questionnaire results}

The questionnaire prepared by the University of Jan Kochanowski in Kielce, Holycross Cancer Center in Kielce and the Polish Society of Medical Physics were sent to more than 20 Polish radiotherapy departments (RD). Seven RDs that routinely use radiotherapy in breast cancer patients after subcutaneous mastectomy with the use of a prosthesis or an expander responded to it, and one RD that performed this procedure several times and had no experience in this field. Therefore, they did not answer the questionnaire directly but sent their comments, which we will quote in full:

1. In planning radiotherapy in such cases, the biggest challenge was obtaining a satisfactory dose in the areas between the implant and the lung/rib (thin layer) and the second the area between the implant and the body surface (essentially skin and subcutaneous tissue/a postoperative scar).

2. The second challenge was the limitation of the treatment planning system, which takes Hounsfield Units (HU) values to 3000 , with the implant material having this parameter much higher. We can measure its $\mathrm{HU}$, but the system does not accept it anyway and overwrites this value.

3. Flares caused by the presence of metal clips cause significant disturbances in $C T$, which can significantly disrupt the dose distribution. The use of density correcting algorithms in $\mathrm{CT}$ is of minimal help.

It is a pity that the other RDs, to which the questionnaire was sent, did not answer as to why they do not routinely use this type of irradiation.

The questionnaire (tab. I) contained questions on formal issues (certificates), treatment planning (beam geometry, normalisation, algorithms), and the values of the total and fraction doses used.

\section{Results}

The values presented in the table show that not all RDs require a manufacturer's certificate, showing that the manufacturer does not prohibit the use of a prosthesis/expander during treatment with ionizing radiation. This is an important aspect of therapy. The lack of a certificate in the medical records can make the legal assessment of treatment very complicated. It would be prudent to require such a certificate before starting treatment. The density of the prosthesis or expander may be much greater than that of the soft tissue. Six RDs declared that the density value was known from the CT examination. The most commonly used irradiation technique is VMAT. Interestingly, none of the radiotherapy departments declared that they (routinely) used the tangential field technique (fig. 1), perhaps they were qualified for the 3DCRT technique).

The most commonly used energy is the X6MV beam. Only one radiotherapy department also uses $\mathrm{X} 15 \mathrm{MV}$ radiation. The 
Table I. Questionnaire with answers. Seven sites responded to the questionnaire, the sum of the points in the question is not always equal to seven, as, for example, different dose fractionation schemes can be used in one site

Questionnaire concerning radiotherapy treatment planning for a breast cancer patient irradiated after a subcutaneous mastectomy with immediate reconstruction with a prosthesis or expander

1. Do you use postoperative radiotherapy in patients after a subcutaneous mastectomy with simultaneous reconstruction?

\begin{tabular}{|l|l|}
\hline yes -6 & no -0 \\
\hline
\end{tabular}

2. Do you use postoperative radiotherapy in patients after a subcutaneous mastectomy with simultaneous reconstruction with the use of an expander?

\begin{tabular}{|l|l}
\hline yes -5 & no -1 \\
\hline
\end{tabular}

3. Do you require the delivery of the prosthesis manufacturer's or expander's certificate for the use of radiotherapy?

\begin{tabular}{|l|l}
\hline yes -5 & no -2
\end{tabular}

4. Do you have the exact value of the prosthesis/expander density resulting from the tomographic examination?

\begin{tabular}{|l|l}
\hline yes -6 & no -1 \\
\hline
\end{tabular}

5. What techniques of radiotherapy do you use in patients irradiated with a prosthesis or an expander?

\begin{tabular}{|l|l|}
\hline 3D-CRT/tangential fields & 2 \\
\hline IMRT & 2 \\
\hline VMAT & 4 \\
\hline tomotherapy & 2 \\
\hline 3D-CRT (field in field) & 1 \\
\hline hybrid 3D-CRT 20\%, VMAT 80\% & 2 \\
\hline
\end{tabular}

6.What kind of radiation do you use in patients irradiated with a prosthesis or an expander?

\begin{tabular}{|l|l|l} 
photons: X6MV - 5 & electrons: 0 & mixed photons: X6MV and X15MV - 1 \\
\hline
\end{tabular}

7. What fraction doses do you use in these patients?

\begin{tabular}{|l|l|l|l|l|}
\hline $1.8 \mathrm{~Gy}-1$ & $2 \mathrm{~Gy}-4$ & $2.25 \mathrm{~Gy}-1$ & $2.5 \mathrm{~Gy}-1$ & $2.67 \mathrm{~Gy}-1$ \\
\hline
\end{tabular}

8. What total doses do you use in these patients?

\begin{tabular}{|l|l|l|l|l|l|}
\hline $45 \mathrm{~Gy}-1$ & $45 \mathrm{~Gy}-1$ & $50 \mathrm{~Gy}-3$ & $50.4 \mathrm{~Gy}-1$ & $40 \mathrm{~Gy}-1$ & $60 \mathrm{~Gy}-1$ \\
\hline
\end{tabular}

\section{How the dose is specified?}

\begin{tabular}{|l|l|}
\hline point & 0 \\
\hline isodose & $2: 95 \% ; 98 \%$ \\
\hline volume & $2: 100 \% ; 95 \%$ PTV \\
\hline mean & 4 \\
\hline minimum & 0 \\
\hline other & raport ICRU 83 \\
\hline
\end{tabular}

10. How is the patient positioned?

\begin{tabular}{l|l|}
\hline supine position -6 & srone position -1 \\
\begin{tabular}{|l|l|}
\hline 11. Do you use skin bolus in these patients? & no -5 \\
\hline yes -2 &
\end{tabular}
\end{tabular}

12. What calculation algorithms do you use in these patients?

\begin{tabular}{|l|l|}
\hline CCCS & 1 \\
\hline PBC & 0 \\
\hline AAA & 4 \\
\hline Acuros & 2 \\
\hline Monte Carlo & 1 \\
\hline other & 0 \\
\hline
\end{tabular}

13. At what distances are computed tomography scans?: $1.5 ; 2$ and $3[\mathrm{~mm}]$

\begin{tabular}{|c|c|c|}
\hline $1,5 \mathrm{~mm}-1$ & $2,5 \mathrm{~mm}-2$ & $3 \mathrm{~mm}-5$ \\
\hline
\end{tabular}

14. What value of the calculation grid is used in the dose calculation?: $2.5 ; 3 ; 5$ [mm]
$2,5 \mathrm{~mm}-5$
$3 \mathrm{~mm}-2$

15. Please attach prepared DVH for treated patient 


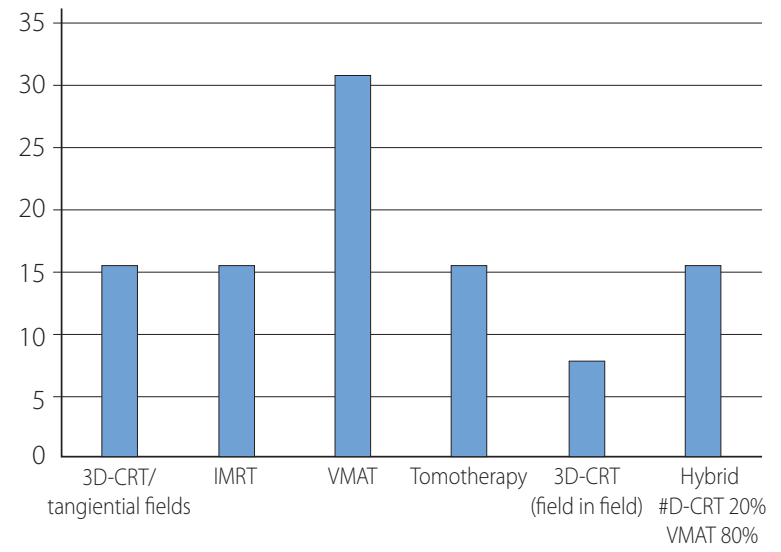

Figure 1. Percentage of irradiation techniques in patients treated for breast cancer after a subcutaneous mastectomy with the use of a prosthesis or expander in seven Polish radiotherapy departments

planned total and fraction doses are in the range from 40 to $60 \mathrm{~Gy}$ and from 2 to $2.67 \mathrm{~Gy}$, respectively. The spread is quite significant. If we add to this that these doses are normalised to the mean value (57\% of RDs), the remaining ones to the volume (100\% or $95 \%$ ) and dose ( $98 \%$ or $95 \%$ ), it may turn out that despite the records in the treatment card in different RDs are the same, but in reality, they differ significantly [8].

The algorithms used to calculate the doses take into account "almost" all the physical phenomena related to radiation absorption. None of the RDs declared that they used the Pencil Beam algorithm [9]. Therefore, it is very likely that the obtained calculation results are consistent with the actual doses, of course within the limits of measurement uncertainty. However, it should be remembered that the results refer to absorbed energy, i.e. a physical phenomenon. They do not take into account the change in the energy spectrum that may occur on the border of two densities and which affects the biological effects. This is one reason to be very careful when planning when there is an "object" of high density within the delivery volume. Computed tomography is performed with distances between the layers from 1.5 to $5 \mathrm{~mm}$ and the computational grid from 2.5 to $5 \mathrm{~mm}$. The questionnaire also asked for a sample DVH. It is difficult to discuss and compare dose values on DVH charts when the planned total doses are different. Therefore, it is worth looking at the anatomical structures that are analysed. All the graphs show: PTV, heart, lungs: right and left and their sum, but only one has an "expander". This outline is introduced not to know what doses are in it, but to exclude it from the PTV volume because it distorts the information about its distribution.

Since the information that we obtained from the questionnaire speaks about differences in dose definition and data acquisition from a CT scanner, it is worth analysing how large the differences in doses may be.

\section{Discussion}

\section{The total dose, fractional dose and the method of its normalisation}

Figure 2 shows an example of the dose distribution with an expander. Most of the valves used are less than $3000 \mathrm{HU}$ and have no significant effect on the dose distributions.

The most frequently planned fraction dose is $2 \mathrm{~Gy}$, and the total 50 Gy normalised to the mean dose value in PTV (fig. 3). The differences between dose distributions when normalised to a mean value or 95\% dose in 95\% PTV volume are negligible, both in PTV/CTV and critical structures.

Fraction and total doses may have a much greater impact on the results of treatment. Let us assume a / $\beta=4 \mathrm{~Gy}$, let us assume the administration of a fractionated dose of $50 \mathrm{~Gy}$ at 2 Gy and 2.5 Gy. The biologically equivalent doses (DizoGy2)

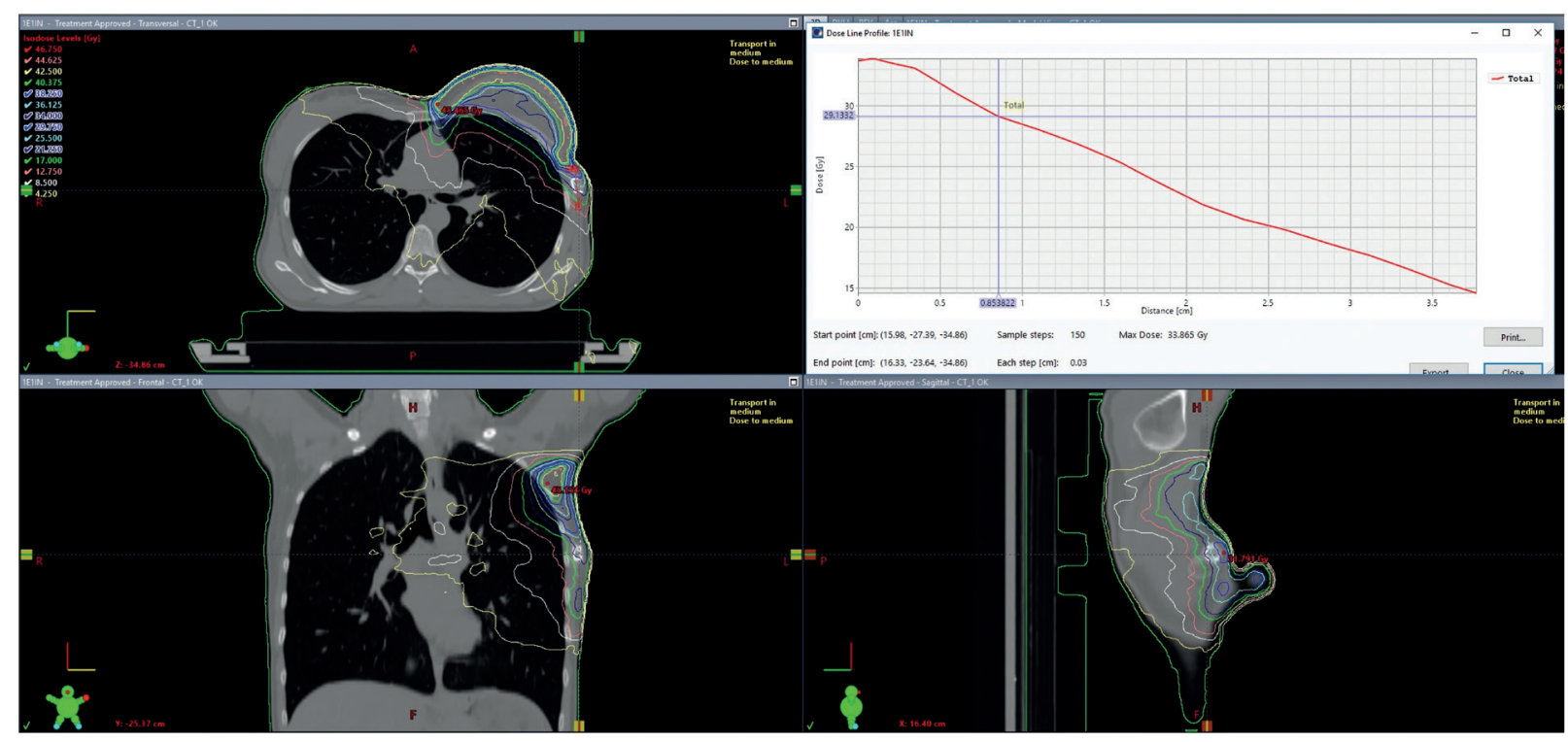

Figure 2. An example of dose distribution with an expander 


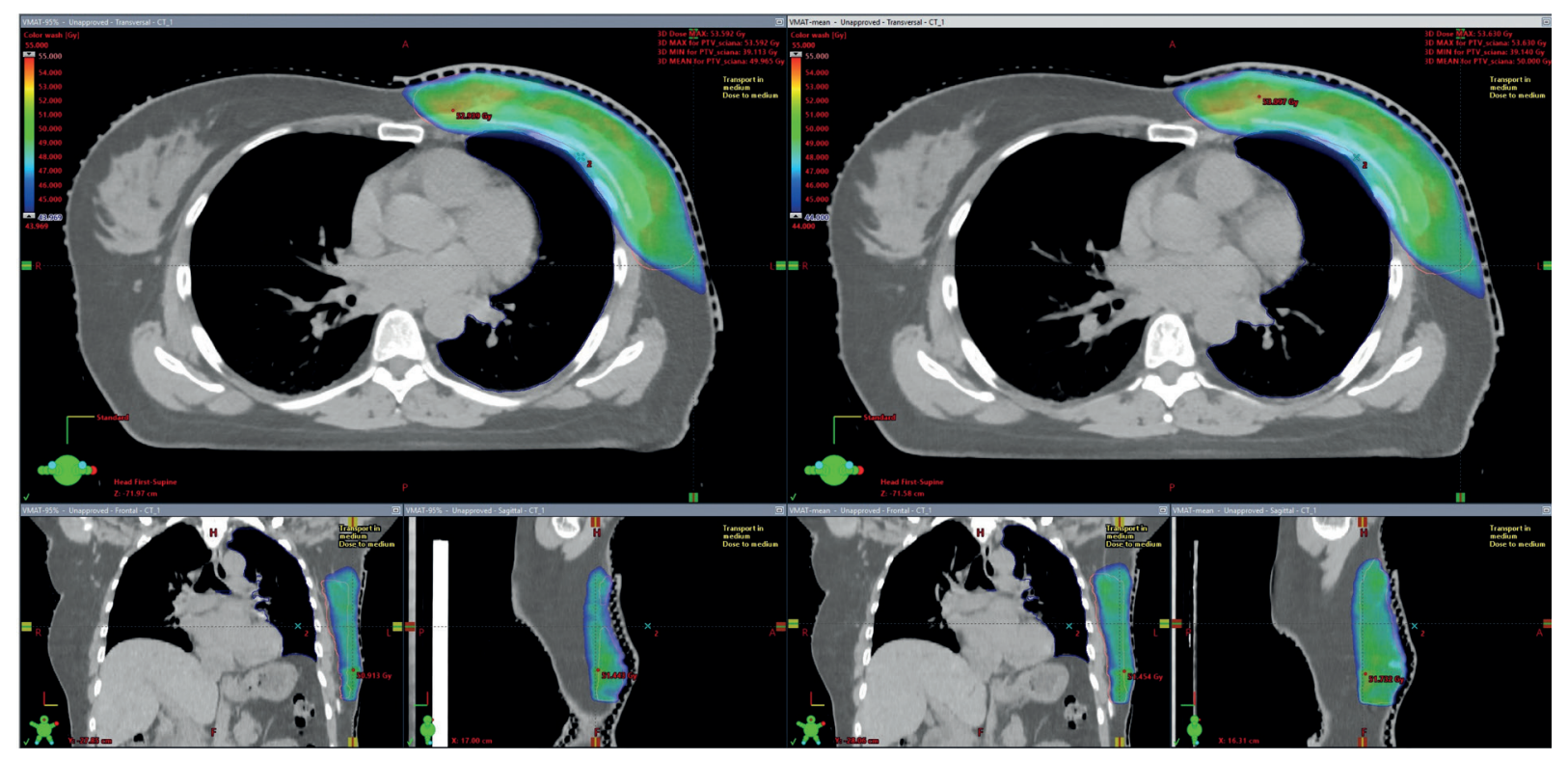

Figure 3. An example of 50 Gy total dose distribution in PTV. Normalisation to $95 \%$ of the dose in $95 \%$ PTV (A) and the mean value in PTV (B), $2.5 \mathrm{~mm}$ dose grid, Acuros v 15.6 algorithm (VMS). In this case, the values of the maximum, minimum, and average doses differ by no more than 0.2 Gy

will be 50 Gy and 54.2 Gy. These differences may be important in the assessment of treatment effects [10].

\section{Effect of the calculation grid on the results of the dose distribution}

Dose distributions were calculated for the calculation grid: $1 \mathrm{~mm}$ and $5 \mathrm{~mm}$, the results indicate that there is a difference in the dose distributions, especially in PTV (fig. 4). Significant differences occur in the area of dose escalation (the PTV area under the skin) and on the border: chest wall - lungs which could be important in the analysis of the correlation between the dose and the likelihood of local cure.
There are differences between the calculations performed by different algorithms. However, they are difficult to estimate based on this questionnaire. Since these are algorithms that take into consideration most of the physical phenomena, including the boundary effects related to the difference in density, it can be assumed that the calculated dose distributions are consistent with the measurement results. The questionnaire did not include the question of whether the dose distribution was verified in the case of dynamic techniques before starting treatment. As this is a formal requirement, we recognise that all centres comply with the applicable regulations.



Figure 4. DVH for PTV for dose grid of 5 (A) and 1 (B) mm (AAA algorithm, eclipse, VMS). The differences between the minimum, maximum, and average doses are in the order of $1 \mathrm{~Gy}$, the values are higher for a smaller dose grid 


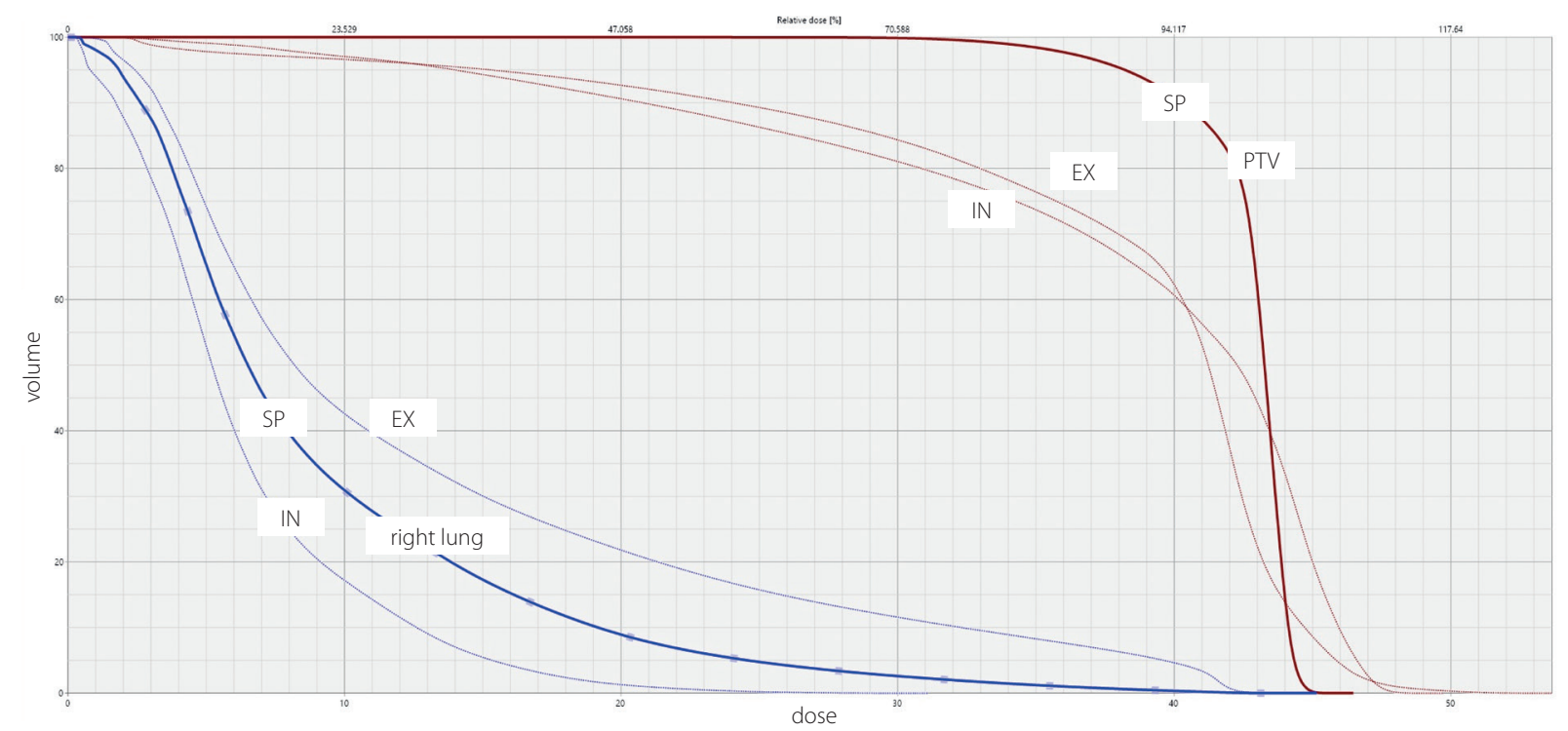

Figure 5. The simulation of chest movement associated with breathing (EX - exhale, IN - inhale, SP - stable, planned position) has a significant impact on dose distributions (without respiratory gating)

An important aspect of chest area irradiation is respiratory mobility. Figure 5 shows the DVH simulating respiratory mobility. It seems that in this anatomical location it is justified to use the respiratory gating technique (especially full inspiration) or tracking the location of the irradiated area-tracking target $[11,12]$.

A subcutaneous mastectomy with immediate reconstruction with an expander or prosthesis is becoming more common and sometimes replaces breast-conserving surgery in the hope that radiotherapy is not necessary. However, irradiation should be used in many patients after subcutaneous mastectomy. A very thorough diagnostic workup is demanded and proper qualification for this surgical procedure. This is also a challenge for radiation oncologists and medical physicists. Prospective trials are necessary to ensure that these new techniques do not compromise oncologic outcomes[13-16].

\section{Conclusions}

The greatest differences in irradiation and irradiation planning in patients treated for breast cancer after a subcutaneous mastectomy with the use of a prosthesis or expander between radiotherapy departments concern total and fraction doses. The irradiation techniques are mainly dynamic techniques (VMAT) and the algorithms used take into account most of the physical phenomena related to radiation absorption. The methods of normalisation do not make any significant differences in the dose distributions. The position of the patient is very similar across all RDs. Most radiotherapy departments require an expander or prosthesis with a manufacturer's certificate. This is an important aspect from a formal point of view. Most expander valves have a slightly higher density than bone. However, differences in chemical composition must be taken into consideration. They do not significantly affect the dose distributions, however, attention should be paid to the disturbances that are introduced. The actual problem is usually the lack of information from the manufacturer as to whether the material can be used in radiotherapy. We do not know if its properties change as a function of the absorbed dose. When preparing patients for irradiation, in particular when performing computed tomography, the examination protocol should be selected to minimise artifacts that may appear. Due to the mobility of this area, related to the patient's breathing. The use of respiratory gating, despite the prolongation of the therapeutic session, seems to be justified.

\section{Conflict of interest: none declared}

\section{Piotr Kędzierawski}

Jan Kochanowski University

Collegium Medicum

Institute of the Health Sciences

ul. Żeromskiego 5

25-369 Kielce, Poland

e-mail:piotrkedzierawski@wp.pl

Received: 29 Sep 2020

Accepted: 4 Nov 2020

\section{References}

1. Effect of radiotherapy after breast-conserving surgery on 10-year recurrence and 15-year breast cancer death: meta-analysis of individual patient data for 10801 women in 17 randomised trials. Lancet. 2011; 378(9804): 1707-1716, doi: 10.1016/s0140-6736(11)61629-2.

2. Clarke M, Collins R, Darby S, et al. Early Breast Cancer Trialists'Collaborative Group (EBCTCG). Effects of radiotherapy and of differences in the extent of surgery for early breast cancer on local recurrence and 15-year survival: an overview of the randomised trials. Lancet. 2005; 366(9503): 2087-2106, doi: 10.1016/S0140-6736(05)67887-7, indexed in Pubmed: 16360786.

3. Van der Giessen PH. Measurement of the peripheral dose for the tangential breast treatment technique with Co-60 gamma radiation and high energy X-rays. Radiother Oncol. 1997; 42(3): 257-264, doi: 10.1016/ s0167-8140(96)01884-1, indexed in Pubmed: 9155075. 
4. Bree Nv, Battum LJv, Huizenga $\mathrm{H}$, et al. Three-dimensional dose distribution of tangential breast treatment: a national dosimetry intercomparison. Radiother Oncol. 1991; 22(4): 252-260, doi: 10.1016/01678140(91)90159-e.

5. Mukesh MB, Barnett GC, Wilkinson JS, et al. Randomized controlled trial of intensity-modulated radiotherapy for early breast cancer: 5 -year results confirm superior overall cosmesis. J Clin Oncol. 2013;31(36):4488-4495, doi: 10.1200/JCO.2013.49.7842, indexed in Pubmed: 24043742.

6. Karpf D, Sakka M, Metzger M, et al. Left breast irradiation with tangential intensity modulated radiotherapy (t-IMRT) versus tangential volumetric modulated arc therapy (t-VMAT): trade-offs between secondary cancer induction risk and optimal target coverage. Radiat Oncol. 2019; 14(1): 156, doi: 10.1186/s13014-019-1363-4, indexed in Pubmed: 31477165.

7. Agha RA, Al Omran Y, Wellstead G, et al. Nipple sparing versus skin sparing mastectomy: a systematic review protocol. BMJ Open. 2016; 6(5): e010151-145, doi: 10.1136/bmjopen-2015-010151, indexed in Pubmed: 27207622.

8. Ślosarek K, Kopczyńska J, Osewski W. Dose specification in External Beam Radiotherapy for CyberKnife and VMAT techniques applied to a case of prostate cancer. Nowotwory. Journal of Oncology. 2017;66(5): 375-380, doi: 10.5603/njo.2016.0067.

9. Knöös T, Ceberg C, Weber L, et al. The dosimetric verification of a pencil beam based treatment planning system. Phys Med Biol. 1994; 39(10): 1609_ 1628, doi: 10.1088/0031-9155/39/10/007, indexed in Pubmed: 15551534.

10. Cefaro G, Genovesi D, Perez C. Delineating Organs at Risk in Radiation Therapy. 2013, doi: 10.1007/978-88-470-5257-4.

11. Dumane VA, Saksornchai K, Zhou Y, et al. Reduction in low-dose to normal tissue with the addition of deep inspiration breath hold (DIBH) to volumetric modulated arc therapy (VMAT) in breast cancer patients with implant reconstruction receiving regional nodal irradiation. Radiat Oncol. 2018; 13(1): 187, doi: 10.1186/s13014-018-1132-9, indexed in Pubmed: 30249274.

12. Testolin A, Ciccarelli S, Vidano G, et al. Deep inspiration breath-hold intensity modulated radiation therapy in a large clinical series of 239 left-sided breast cancer patients: a dosimetric analysis of organs at risk doses and clinical feasibility from a single center experience. $\mathrm{Br} J$ Radiol. 2019; 92(1101): 20190150, doi: 10.1259/bjr.20190150, indexed in Pubmed: 31265316.

13. Mitchell MP, Wagner J, Butterworth J. Subcutaneous implant-based breast reconstruction, a modern challenge in postmastectomy radiation planning. Pract Radiat Oncol. 2018; 8(3): 153-156, doi: 10.1016/j. prro.2017.09.001, indexed in Pubmed: 29233522.

14. Zheng $\mathrm{Y}$, Zhong $\mathrm{M}, \mathrm{Ni}$, et al. Radiotherapy and nipple-areolar complex necrosis after nipple-sparing mastectomy: a systematic review and meta-analysis. Radiol Med. 2017; 122(3): 171-178, doi: 10.1007/ s11547-016-0702-x, indexed in Pubmed: 28000160.

15. Ben-David M, Granot H, Gelernter I, et al. Immediate breast reconstruction with anatomical implants following mastectomy: The radiation perspective. Med Dosim. 2016; 41(2): 142-147, doi: 10.1016/j.meddos.2015.11.002, indexed in Pubmed: 26923467.

16. Leonardi MC, Spoto R, Miglietta E, et al. HALFMOON TomoTherapy (Helical ALtered Fractionation for iMplant partial OmissiON): implant-sparing post-mastectomy radiotherapy reshaping the clinical target volume in the reconstructed breast. J Cancer Res Clin Oncol. 2019; 145(7): 1887-1896, doi: 10.1007/s00432-019-02938-8, indexed in Pubmed: 31144158. 